\title{
Heat and Mass Transfer of a Peristaltic Electro-osmotic Flow of a Couple Stress Fluid through an Inclined Asymmetric Channel with Effects of Thermal Radiation and Chemical Reaction
}

\author{
Kattamreddy Venugopal Reddy', Machireddy Gnaneswara Reddy², Oluwole Daniel Makinde ${ }^{3}$ \\ 1 Division of Mathematics, Department of Sciences and Humanities, Faculty of Science, Vignan Institute of Technology and \\ Science, 508284 Deshmukhi, Telangana, India \\ 2 Department of Mathematics, Faculty of Mathematics, Acharya Nagarjuna University, Ramnagar, 523001 Ongole, Andhra \\ Pradesh, India \\ ${ }^{3}$ Department of Mathematics, Faculty of Military Science, Stellenbosch University, 7395 Saldanha, Private Bag X2, South Africa \\ ${ }^{*}$ Corresponding author, e-mail: venugopal.reddy1982@gmail.com
}

Received: 30 June 2020, Accepted: 30 November 2020, Published online: 16 March 2021

\begin{abstract}
The presented article addresses the electro-osmotic peristaltic flow of a couple stress fluid bounded in an inclined asymmetric microchannel. The viscous dissipation, Joule heating and chemical reaction effects are employed simultaneously in the flow analysis. Heat and mass transfer have been studied under large wavelength and small Reynolds number. The resulting nonlinear systems are solved numerically. The influence of various dominant physical parameters is discussed for velocity, temperature distribution, concentration distribution and the pumping characteristics. Electro kinetic flow of fluids by micro-pumping through micro channels and micro peristaltic transport has accelerated considerable concern in accelerated medical technology and several areas of biomedical engineering. Deeper clarification of the fluid dynamics of such flow requires the continuous need for more delicate mathematical models and numerical simulations, in parallel with laboratory investigations.
\end{abstract}

Keywords

peristaltic flow, electro-osmotic flow, couple stress fluid, magnetic field, heat transfer, mass transfer, inclined asymmetric micro-channel

\section{Introduction}

Recent investigations in miniaturization and micro-fabrication have taken into assuming a lot of applications extending from organic to refrigerating of microelectronics in [1-9]. Many favors such as an important contraction in the utilization of prescribed materials, ability to achieve on the continuous motion of in-vitro experiments in a aspect similar to the absolute situation in a biological living system, being vibration-free and portable are using microfluidic devices. Electro-osmotic transports with thermal effects of liquids in micro channels are reported in [10]. The transfer of heat investigation of osmotic electro motion in a slowly varying non-symmetric micro-channel is presented in [11].

Stokes matured the model of non-Newtonian couple stress fluid. An additives are alloyed in the fluid then united forces of fluid abide additive factors. This resistance builds a mixed force and then a non-Newtonian couple stresses is made in the fluid. This fluid is called as non-Newtonian couple stress fluid. Such a model is observed as rationalization of Newtonian fluid model trading with couple stresses and body couples in fluid channel. It is important that stress tensor involves couple stress fluid. Significant studies in this order are made in the findings [12-18].

Bio-fluids send from one region to another region by unceasing process of muscle relaxation and contraction. This mechanism is called as peristaltic movement. This phenomenon is important for the flow of biological fluids in a few physiological action such as urine flow to bladder through kidney, the transport of chyme into the gastrointestinal tract, fluids in the lymphatic vessels, bile from the gallbladder into the duodenum, the embryo movement in non-pregnant uterus, the transport of spermatozoa in the ducts eccentric of the male reproductive tract, the movement of the ovum in the fallopian tube and the dissemination of blood in small blood vessels are presented in [19-37]. 
Thus the important focus of this study is to investigate the viscous dissipation, Joule heating effects on MHD electro-osmotic peristaltic transport of couple stress fluid in an inclined asymmetric micro channel. Mathematical formulation of problem is an exhibited. The results are obtained after employing long wavelength and low Reynolds number approximation. The velocity, temperature, concentration, pressure gradient and pressure rise have been proposed for the pertinent parameters of interest and also trapping phenomenon has been observed. Electro kinetic flow of fluids by micro-pumping through micro channels and micro peristaltic transport has accelerated considerable concern in accelerated medical technology and several areas of biomedical engineering. Deeper clarification of the fluid dynamics of such flow requires the continuous need for more delicate mathematical models and numerical simulations, in parallel with laboratory investigations.

\section{Methodology}

We analyze the peristaltic electro-osmotic transport of incompressible couple stress fluid which is electrically conducting and transfers of heat through asymmetric inclined micro-channel walls with charged under the impact of a promulgated the magnetic field. The movement is considered to be asymmetric about $X$ and the liquid is flowing in the $X$-direction. The hydrophobic micro-channel is bounded by slowly varying walls at $Y=h_{1}(X)$ and $Y=h_{2}(X)$ respectively, in which the length of the channel $(L)$ is assumed to be much larger than the height, i.e., $L>>\left(h_{1}+h_{2}\right)$.

The geometrical expression of the wavy channels are given by:

$$
\begin{aligned}
& h_{1}^{\prime}(X)=\cos \left(\frac{2 \pi X}{L}\right) a_{1}^{\prime}+d_{1}^{\prime}, \\
& h_{2}^{\prime}(X)=-\cos \left(\frac{2 \pi X}{L}+\phi\right) a_{2}^{\prime}-d_{2}^{\prime} .
\end{aligned}
$$

\subsection{Potential - electrical distribution}

The basic theory of electrostatics is related to the local net electric charge density $\rho_{e}$ in the diffuse layer of EDL and charge density is coupled with the potential distribution $\psi^{\prime}$ through the Poisson-Boltzmann equation for the symmetric electrolyte is given by:

$$
\frac{d^{2} \psi^{\prime}(Y)}{d Y^{2}}=\frac{2 n_{0} e z_{v}}{\varepsilon} \sinh \left(\frac{e z_{v} \psi^{\prime}(Y)}{k_{B} T_{a v}}\right),
$$

where $\eta_{o}$ represents the concentration of ions at the bulk, $\varepsilon$ is the charge of a proton, $z_{v}$ is the valence of ions, $\varepsilon$ is the permittivity of the medium, $k_{B}$ is the Boltzmann constant and $T_{a v}$ is the boundary conditions for potential function are taken as

$\psi^{\prime}(Y)=\psi_{1}^{\prime}$ at $Y=h_{1}^{\prime}(X)$,

$\psi^{\prime}(Y)=\psi_{2}^{\prime}$ at $Y=h_{2}^{\prime}(X)$,

where $\psi_{1}^{\prime}$ and $\psi_{2}^{\prime}$ are the electric potential at the upper and lower wall respectively.

Let us now introduce the following non-dimensional variables,

$$
\left[\psi_{O}, \psi_{1}, \psi_{2}\right]=\frac{e z_{v}}{k_{B} T_{a v}}\left[\psi^{\prime}, \psi_{1}^{\prime}, \psi_{2}^{\prime}\right], \quad y=\frac{Y}{d_{1}^{\prime}} \quad \text { and } \quad x=\frac{X}{L} .
$$

The dimensionless form of Eqs. (1) and (2) and the Poisson-Boltzmann equation defined in Eq. (3) take in the following form,

$$
\begin{aligned}
& h_{1}(x)=\frac{h_{1}^{\prime}}{d_{1}^{\prime}}=1+a \cos (2 \pi x), \\
& h_{2}(x)=\frac{h_{2}^{\prime}}{d_{1}^{\prime}}=-d-b \cos (2 \pi x+\phi),
\end{aligned}
$$

and

$$
\frac{d^{2} \psi_{O}}{d y^{2}}=k^{2} \sinh \left(\psi_{O}\right)
$$

where $a=\frac{a_{1}^{\prime}}{d_{1}^{\prime}}, \quad b=\frac{a_{2}^{\prime}}{d_{1}^{\prime}}, \quad d=\frac{d_{2}^{\prime}}{d_{1}^{\prime}} \quad$ and $k=\frac{d_{1}^{\prime}}{\lambda} \quad$ is defined as the electro-osmotic parameter, $\lambda_{2}$ is the reciprocal of the EDL thickness and is defined by $\frac{1}{\lambda}=\left(\frac{2 n_{o} e^{2} z_{v}^{2}}{\varepsilon k_{B} T_{a v}}\right)^{\frac{1}{2}}$. Thus the electro-osmotic parameter is inversely proportional to EDL thickness $\lambda$. The dimensionless form of boundary conditions defined in Eq. (4) using the dimensionless variables Eq. (5) reduce to

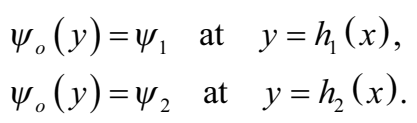

We assumed that the electric potential is much smaller than the thermal potential for which the Debye-Hückel linearization principle can be approximated as $\sinh (x) \approx x$. On the basis of this assumption, the solution of PoissonBoltzmann Eq. (8) takes in the form:

$$
\frac{d^{2} \psi_{o}}{d y^{2}}=k^{2} \psi_{o}
$$


Finally, by employing the boundary conditions Eq. (9), the closed form solution of the Eq. (10) is given as

$\psi_{o}(y)=F_{1} \cosh (k y)+F_{2} \sinh (k y)$.

\subsection{Couple stress fluid model}

The given set of pertinent field equations governing the flow, in laboratory frame is [12, 33]:

$$
\begin{aligned}
& \frac{\partial u^{*}}{\partial x^{*}}+\frac{\partial v^{*}}{\partial y^{*}}=0 \\
& \rho\left(\frac{\partial u^{*}}{\partial t}+u^{*} \frac{\partial u^{*}}{\partial x^{*}}+v^{*} \frac{\partial u^{*}}{\partial y^{*}}\right)=-\frac{\partial P}{\partial x^{*}}+\mu\left(\frac{\partial^{2} u^{*}}{\partial x^{* 2}}+\frac{\partial^{2} u^{*}}{\partial y^{* 2}}\right) \\
& -\eta\left(\frac{\partial^{4} u^{*}}{\partial x^{* 4}}+2 \frac{\partial^{4} u^{*}}{\partial x^{* 2} \partial y^{* 2}}+\frac{\partial^{4} u^{*}}{\partial y^{* 4}}\right)-\sigma B_{0}^{2} u^{*}-\frac{\mu}{k_{o}} u^{*} \\
& +\rho g \beta_{T}\left(T-T_{0}\right) \sin \alpha+\rho g \beta_{C}\left(C-C_{0}\right) \sin \alpha+\rho_{e} E \\
& \rho\left(\frac{\partial v^{*}}{\partial t}+u^{*} \frac{\partial v^{*}}{\partial x^{*}}+v^{*} \frac{\partial v^{*}}{\partial y^{*}}\right)=-\frac{\partial P}{\partial y^{*}}+\mu\left(\frac{\partial^{2} v^{*}}{\partial x^{* 2}}+\frac{\partial^{2} v^{*}}{\partial y^{* 2}}\right) \\
& -\eta\left(\frac{\partial^{4} v^{*}}{\partial x^{* 4}}+2 \frac{\partial^{4} v^{*}}{\partial x^{* 2} \partial y^{* 2}}+\frac{\partial^{4} v^{*}}{\partial y^{* 4}}\right)-\frac{\mu}{k_{o}} v^{*} \\
& +\rho g \beta_{T}\left(T-T_{0}\right) \cos \alpha+\rho g \beta_{C}\left(C-C_{0}\right) \cos \alpha \\
& \rho c_{p}\left(\frac{\partial T}{\partial t}+u^{*} \frac{\partial T}{\partial x^{*}}+v^{*} \frac{\partial T}{\partial y^{*}}\right)=k^{*}\left(\frac{\partial^{2} T}{\partial x^{* 2}}+\frac{\partial^{2} T}{\partial y^{* 2}}\right)+Q_{0} \\
& -\frac{\partial q_{r}}{\partial y^{*}}+\sigma B_{0}^{2} u^{2}+\sigma E^{2} \\
& \left(\frac{\partial C}{\partial t}+u^{*} \frac{\partial C}{\partial x^{*}}+v^{*} \frac{\partial C}{\partial y^{*}}\right)=D\left(\frac{\partial^{2} C}{\partial x^{* 2}}+\frac{\partial^{2} C}{\partial y^{* 2}}\right) \\
& +\frac{D K_{T}}{T_{m}}\left(\frac{\partial^{2} T}{\partial x^{* 2}}+\frac{\partial^{2} T}{\partial y^{* 2}}\right)-k_{1}\left(C-C_{o}\right) .
\end{aligned}
$$

The radiative heat flux in the $X$-direction is considered as negligible compared to $Y$-direction. By using Rosseland approximation for thermal radiation, the radiative heat flux $q_{r}$ is specified by

$$
q_{r}=-\frac{16 \sigma^{*} T_{o}^{3}}{3 k^{*}} \frac{\partial T}{\partial y^{*}},
$$

where $\sigma^{*}$ and $k^{*}$ are the Stefan-Boltzmann constant and the mean absorption coefficient respectively.

The coordinates and velocities in the wave frame $(x, y)$ and the laboratory frame $(X, Y)$ in a coordinate system moving with the wave speed $c$ in which the boundary shape is stationary and are related by $x=x^{*}-c t, y=y^{*}, u=u^{*}-c, v=v^{*}, p(x, y)=P\left(x^{*}, y^{*}, t\right)$, $\bar{T}(x, y)=T\left(x^{*}, y^{*}, t\right), \bar{C}(x, y)=C\left(x^{*}, y^{*}, t\right)$,

where $u, v$ are the velocity components, $p$ is the pressure, $T$ is the temperature and $C$ is the concentration in the wave frame.

We introduce the following non-dimensional quantities:

$\bar{x}=\frac{x}{\lambda}, \bar{y}=\frac{y}{d_{1}}, \bar{u}=\frac{u}{c}, \bar{v}=\frac{v}{c}, h_{1}=\frac{H_{1}}{d_{1}}, h_{2}=\frac{H_{2}}{d_{1}}$,

$\bar{t}=\frac{c t}{\lambda}, \bar{p}=\frac{d_{1}^{2}}{\lambda \mu C} p, \delta=\frac{d_{1}}{\lambda}$,

$d=\frac{d_{2}}{d_{1}}, a=\frac{a_{1}}{d_{1}}, b=\frac{a_{2}}{d_{1}}, \operatorname{Re}=\frac{\rho c d_{1}}{\mu}$,

$M=\sqrt{\frac{\sigma}{\mu}} B_{0} d_{1}, D a=\frac{k_{0}}{d_{1}^{2}}, \gamma=\sqrt{\frac{\mu}{\eta}} d_{1}$,

$\mathrm{Gr}=\frac{\rho g d_{1}^{2} \beta_{T}\left(T_{1}-T_{0}\right)}{\mu C}, \bar{\psi}=\frac{\psi}{c d_{1}}, \operatorname{Pr}=\frac{\mu c_{p}}{k^{*}}$,

$\theta=\frac{\bar{T}-\bar{T}_{0}}{\bar{T}_{1}-\bar{T}_{0}}, \beta=\frac{Q_{0} d_{1}^{2}}{k^{*}\left(\bar{T}_{1}-\bar{T}_{0}\right)}$,

$G c=\frac{\rho g d_{1}^{2} \beta_{C}\left(\bar{C}_{1}-\bar{C}_{0}\right)}{\mu C}, \phi=\frac{\bar{C}-\bar{C}_{0}}{\bar{C}_{1}-\bar{C}_{0}}$,

$S c=\frac{\mu}{\rho D}, S r=\frac{\rho D K_{T}\left(\bar{T}_{1}-\bar{T}_{0}\right)}{\mu T_{m}\left(\bar{C}_{1}-\bar{C}_{0}\right)}$,

$\gamma_{1}=k_{1} \frac{d_{1}^{2}}{v}, R d=\frac{16 \sigma^{*} T_{o}^{3}}{3 k^{*} \bar{\mu}_{o} c_{f}}, C_{o}=\frac{\left(-\frac{d \bar{p}}{d \bar{X}}\right) d_{1}^{2}}{\mu U_{H S}}$.

Using the above transformations Eqs. (17) and (18) and non-dimensional quantities Eq. (19), the governing flow field equations Eqs. (13) to (15), after dropping the bars, we get the dimensional equations of the couple stress fluid are:

$$
\begin{aligned}
& \frac{\partial^{4} \psi}{\partial y^{4}}-\frac{1}{\gamma^{2}} \frac{\partial^{6} \psi}{\partial y^{6}}-\left(M^{2}+\frac{1}{D a}\right) \frac{\partial^{2} \psi}{\partial y^{2}}+\frac{d^{3} \psi_{o}}{d y^{3}} \\
& +\operatorname{Gr} \frac{\partial \theta}{\partial y} \sin \alpha+G c \frac{\partial \phi}{\partial y} \sin \alpha=0 \\
& \frac{(1+R d)}{\operatorname{Pr}} \frac{\partial^{2} \theta}{\partial y^{2}}+E c\left[\left(\frac{\partial^{2} \psi}{\partial y^{2}}\right)^{2}+\frac{1}{\gamma^{2}}\left(\frac{\partial^{3} \psi}{\partial y^{3}}\right)^{2}\right] \\
& +E c M^{2}\left(\frac{\partial \psi}{\partial y}\right)^{2}+A=0 \\
& \frac{\partial^{2} \phi}{\partial y^{2}}-S c \gamma_{1} \phi+S c S r \frac{\partial^{2} \theta}{\partial y^{2}}=0 .
\end{aligned}
$$


The corresponding boundary conditions are:

$$
\psi=\frac{F}{2}, \frac{\partial \psi}{\partial y}+L \frac{\partial^{2} \psi}{\partial y^{2}}=-1, \frac{\partial^{3} \psi}{\partial y^{3}}=0, \theta=0, \phi=0
$$

at

$y=h_{1}=1+a \cos (2 \pi x)$,

$\psi=-\frac{F}{2}, \frac{\partial \psi}{\partial y}-L \frac{\partial^{2} \psi}{\partial y^{2}}=-1, \frac{\partial^{3} \psi}{\partial y^{3}}=0, \theta=1, \phi=1$

at

$y=h_{2}=-d-b \cos (2 \pi x+\phi)$,

where $L$ is the velocity slip parameter and $F$ is the flux in the wave frame and the constants $a, b, \phi$ and $d$ should satisfy the relation

$a^{2}+b^{2}+2 a b \cos \phi \leq(1+d)^{2}$.

The dimensionless mean flow rate $\Theta$ in the fixed frame is related to the non-dimensional mean flow rate $F$ in wave frame by:

$\Theta=F+1+d$,

and in which:

$F=\int_{h_{1}}^{h_{2}} \frac{\partial \psi}{\partial y} \mathrm{~d} y$.

The Nusselt number and Sherwood number are presented in Eqs. (28) and (29):

$$
\begin{aligned}
& \left.\mathrm{Nu}=-\frac{\partial \theta}{\partial y}\right]_{y=h_{1}, h_{2}}, \\
& \left.\mathrm{Sh}=-\frac{\partial \phi}{\partial y}\right]_{y=h_{1}, h_{2}} .
\end{aligned}
$$

\section{Numerical solution}

The solution of system of coupled non-linear Eqs. (20) to (22) with corresponding boundary conditions in Eqs. (23) and (24) are obtained using NDSolve in Mathematica computational software.

This section contains the plots and related analyses for different embedded parameters. This section includes the graphs for velocity distribution, temperature distribution, concentration distribution, pumping characteristics, heat transfer coefficient, mass transfer coefficient and trapping phenomenon.

\subsection{Velocity distribution}

Fig. 1 presents the illustrative diagram of the problem under present study. Fig. 2 displays the velocity profile for various values of Hartmann number. The velocity decreases and drops with Hartmann number $M$. Fig. 3 depicts velocity profiles of different values of parameter for osmosis parameter. Velocity profile is seen to raise as osmosis parameter $k$ enlarges. Fig. 4 depicts that the consequences of the parameter $\lambda_{1}$ on the profile of velocity. It is clear that enhance the strength of $\lambda_{1}$ resulted in enhancing the velocity. Fig. 5 indicates that the velocity rises with increasing values of $D a$. Fig. 6 reveals that velocity is seen to decrease with the higher values of couple stress parameter. Fig. 7 shows that velocity diminishes with increasing values of $L$.

\subsection{Temperature distribution}

Figs. 8 to 11 depict the deviations in temperature profiles for various values of parameters $A, k, R d$ and $E c$. Fig. 8 presents the consequences of the parameter $A$ on the

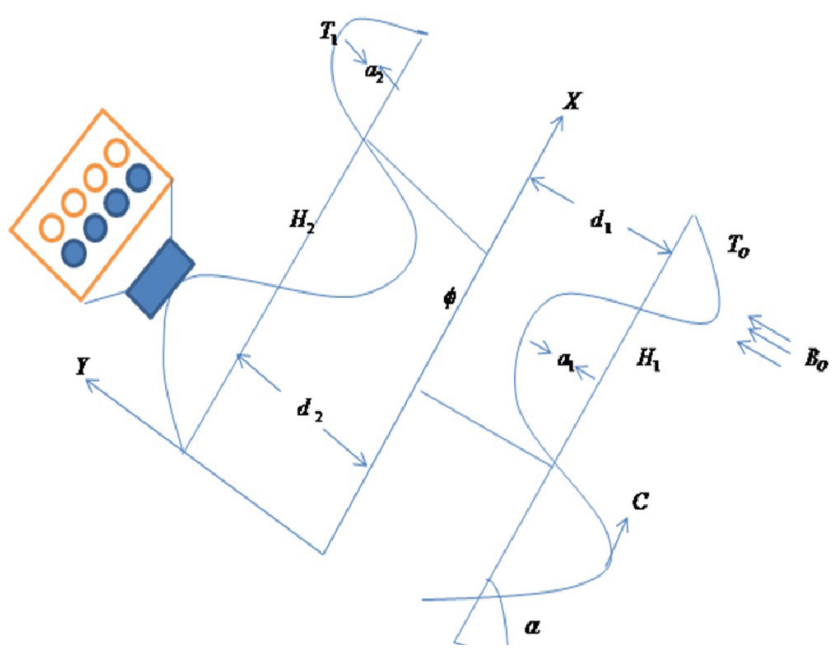

Fig. 1 Physical model

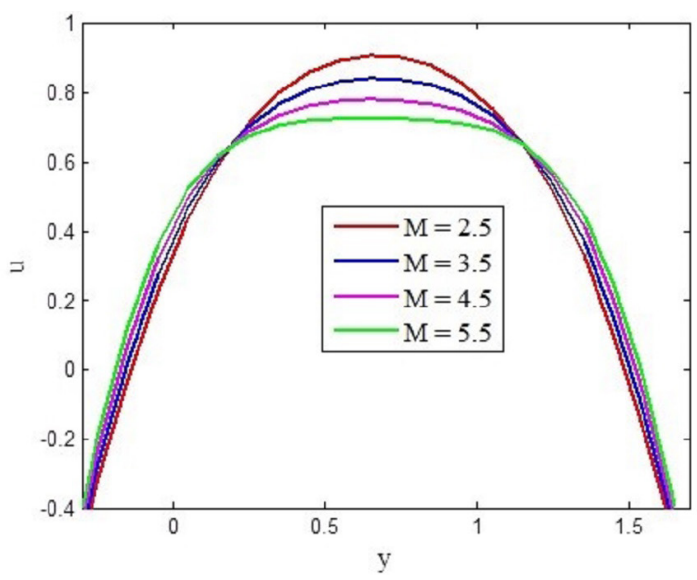

Fig. $2 u$ for $M$ 


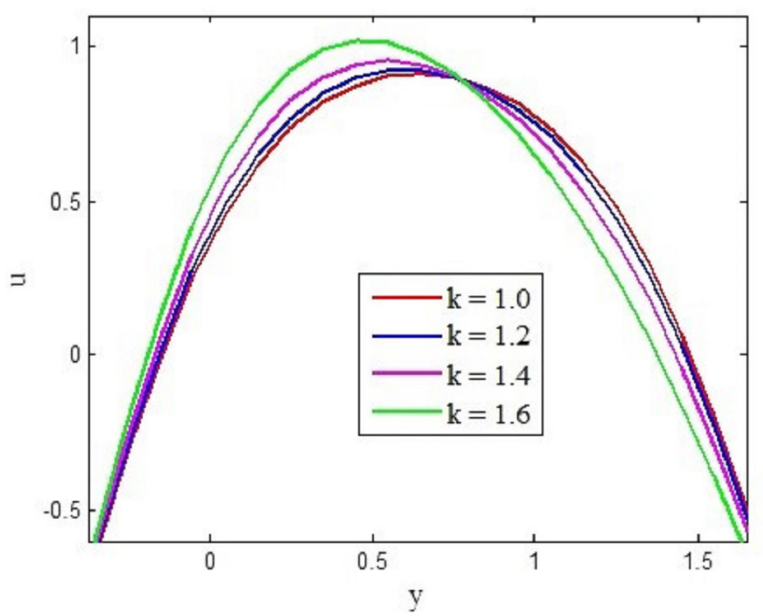

Fig. $3 u$ for $k$

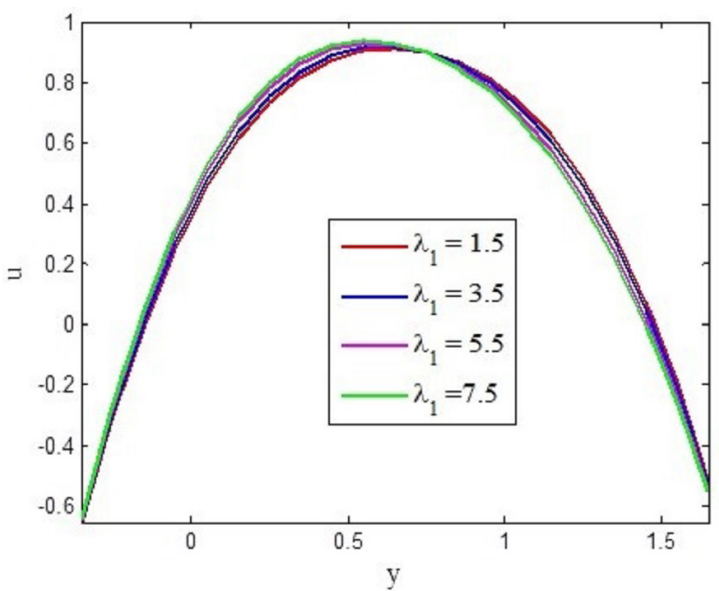

Fig. $4 u$ for $\lambda_{1}$

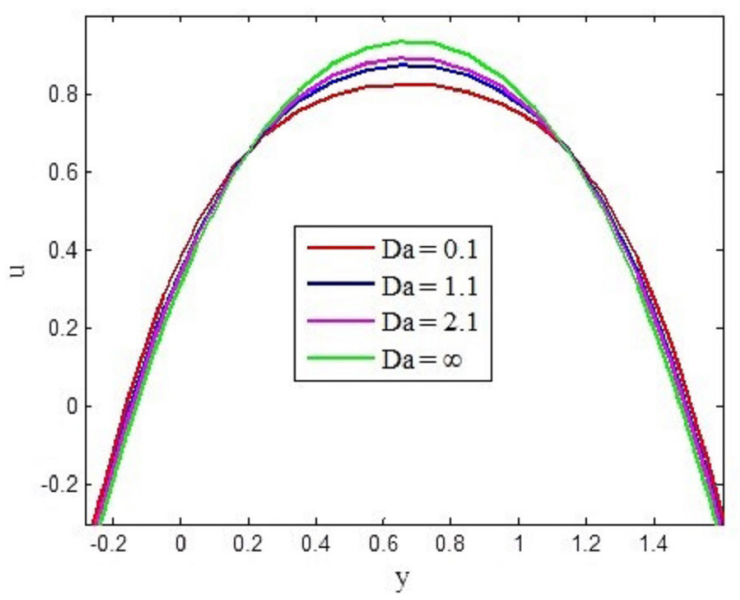

Fig. $5 u$ for $D a$

profile of temperature. It is clear that enhance the strength of $A$ resulted in increasing the temperature. Fig. 9 shows that temperature rises with enhancing $k$. Fig. 10 shows that the temperature diminishes significantly with a rise in $R d$ whereas the temperature increases as the higher values of $E c$ from Fig. 11.

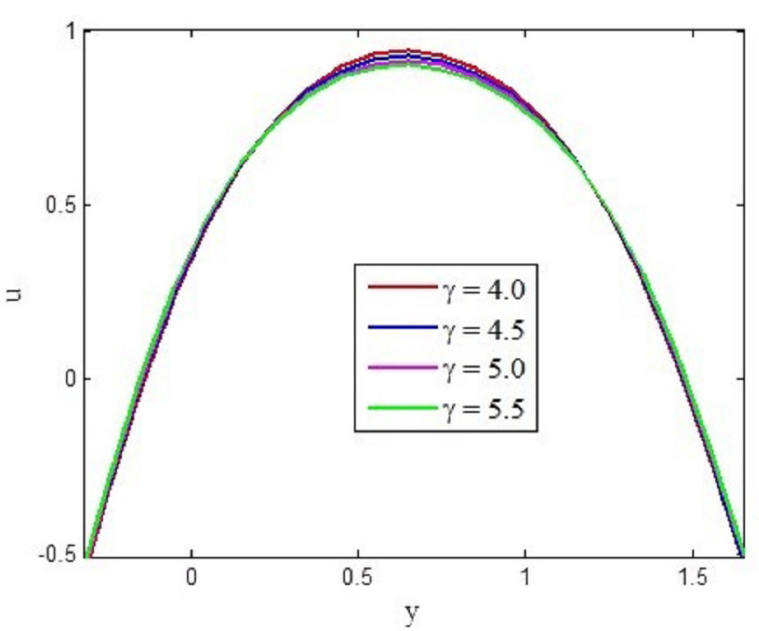

Fig. $6 u$ for $\gamma$

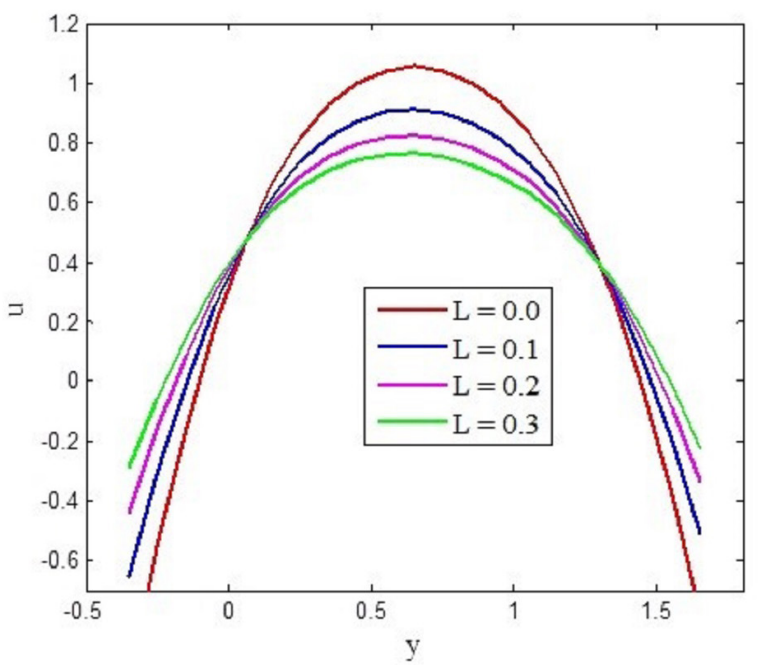

Fig. $7 u$ for $L$

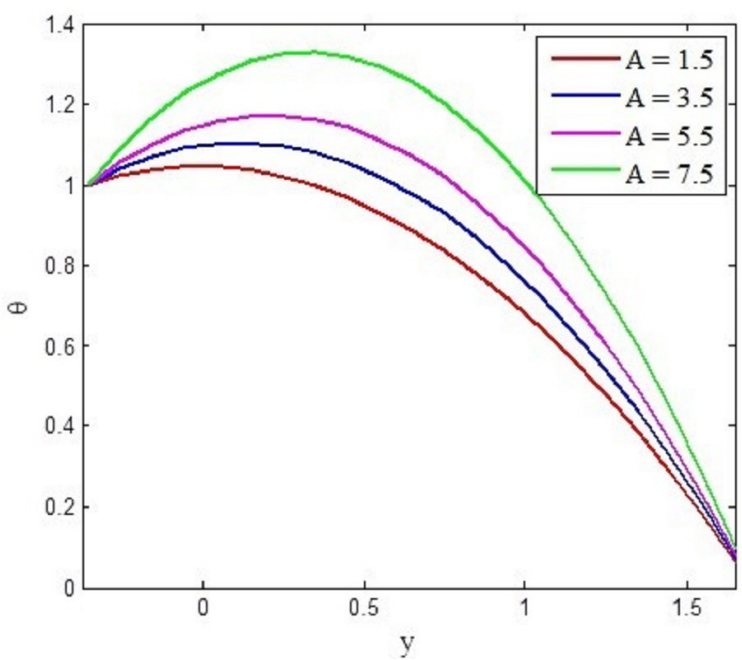

Fig. $8 \theta$ for $A$

Fig. 12 shows a very significant effect of Pr on the temperature profiles. It is clear from Fig. 12 that the Brinkman number has an impulse to diminish the temperature in the 


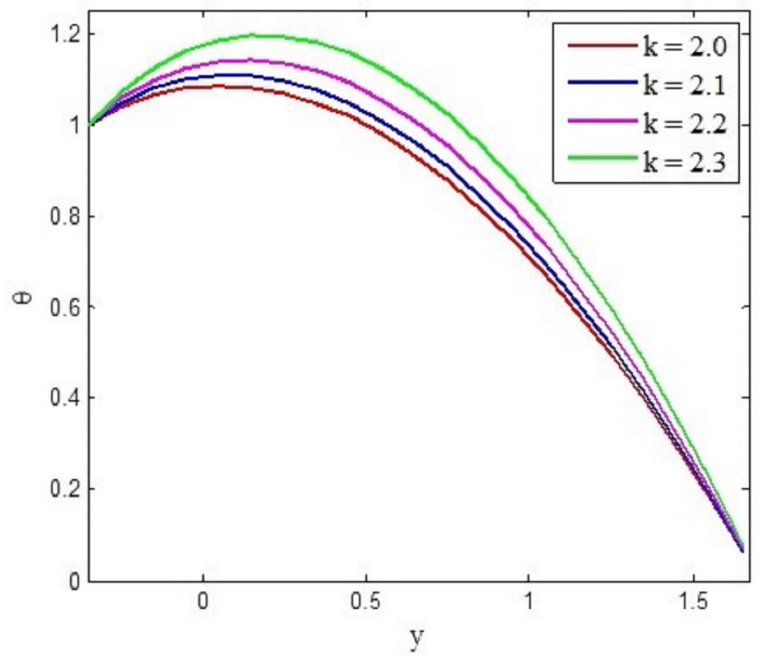

Fig. $9 \theta$ for $k$

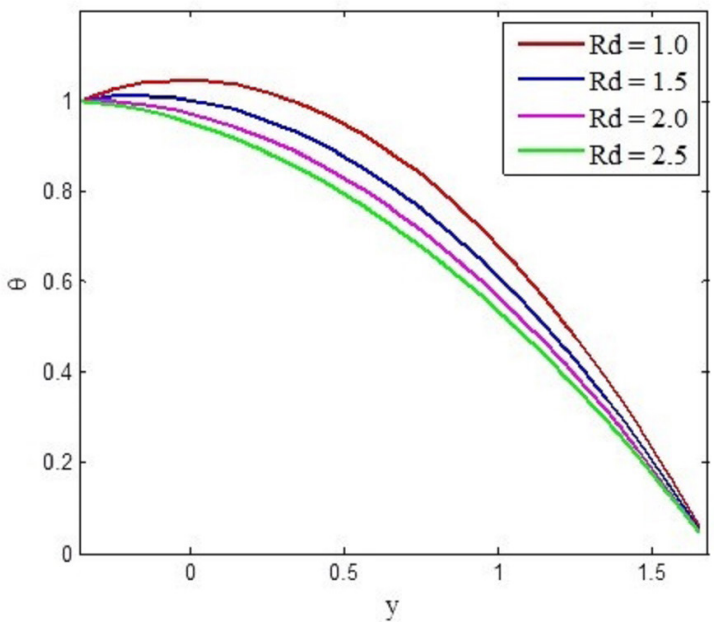

Fig. $10 \theta$ for $R d$

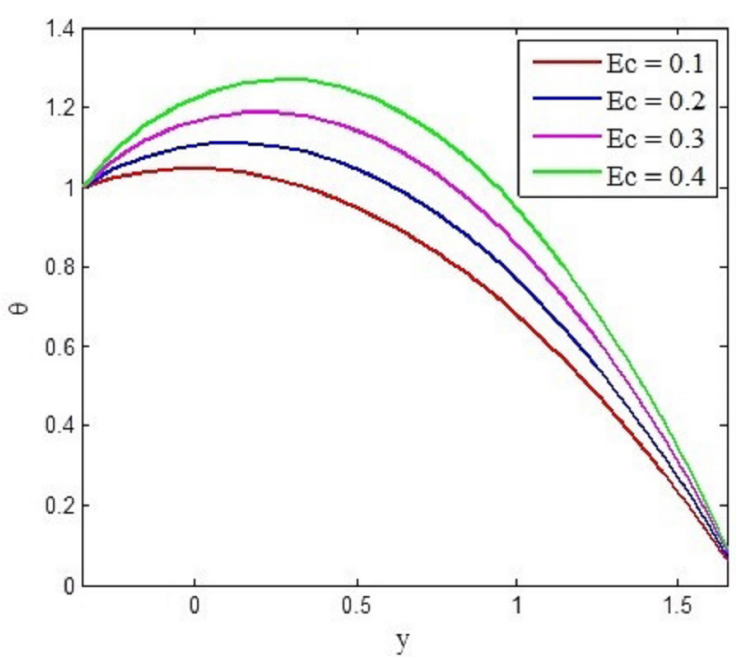

Fig. $11 \theta$ for $E c$

micro-channel. It may be offered that the conductivity of thermal of the fluid drops by increasing the ratio of the diffusivity of the momentum to diffusivity of the thermal.

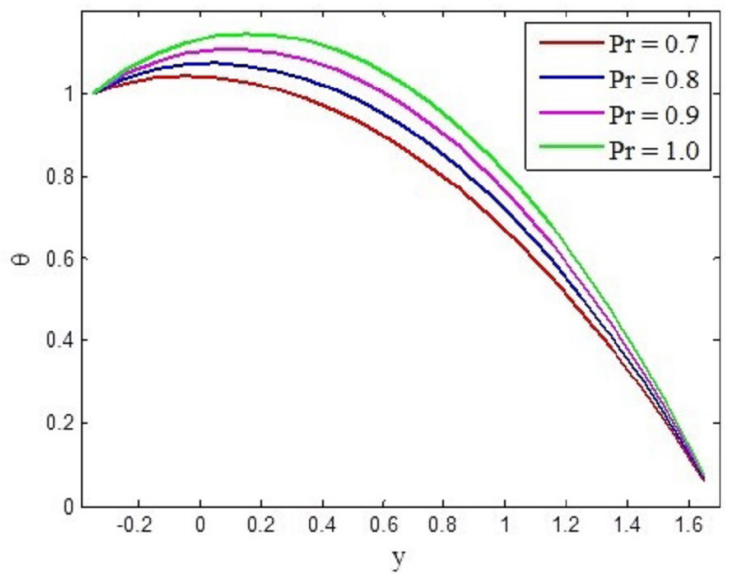

Fig. $12 \theta$ for $\operatorname{Pr}$

\subsection{Concentration distribution}

Figs. 13 to 15 present the consequences of the parameter $S c, S r$ and $\gamma_{1}$ on the profile of concentration. It is clear that enhance the strength of $S c$ resulted in diminishing the concentration in Fig. 13. Fig 14 depicts that the

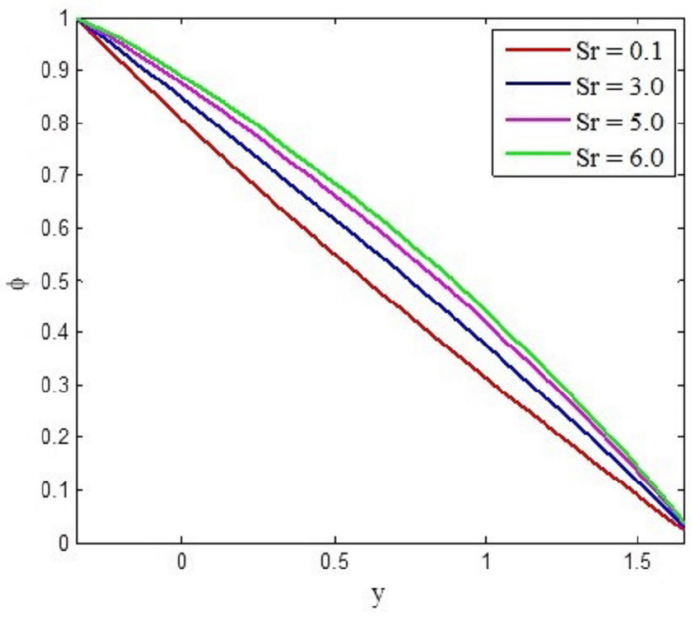

Fig. $13 \phi$ for $S r$

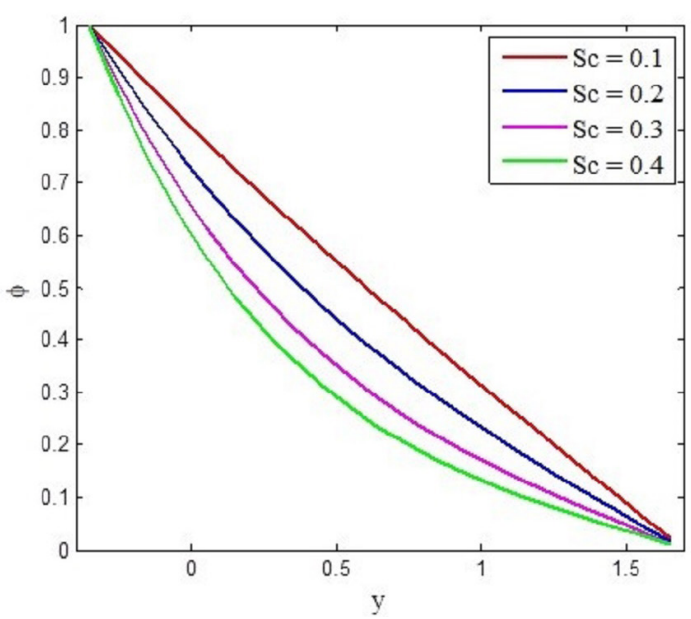

Fig. $14 \phi$ for $S c$ 


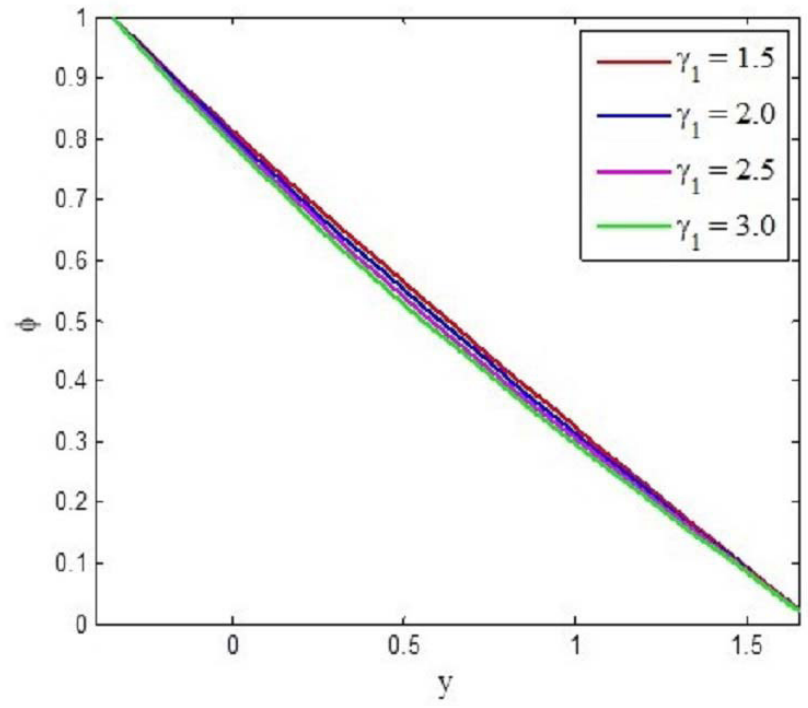

Fig. $15 \phi$ for $\gamma_{1}$

concentration enhances with an increasing the values of $S r$. Fig 15 reveals that the concentration is seen to decrease with the enhancing the values of $\gamma_{1}$.

\subsection{Pumping characteristics}

Figs. 16 to 18 represent the profiles of pressure gradient $\left(\frac{d p}{d x}\right)$ for the effects of Slip parameter $(L)$, Osmosis parameter $(k)$ and the couple stress parameter $(\gamma)$. The pressure gradient has oscillatory behavior in the whole range of the $x$-axis. From all figures, it is clear that the pressure gradient diminishes with the higher values of $L, k$ and $\gamma$.

The pressure rise is a significant physical measure in the peristaltic mechanism. The results are prepared and discussed for different physical parameters of interest through Figs. 19 to 22 and which are plotted for dimensionless pressure rise $\Delta P_{\lambda}$ versus the dimensionless flow

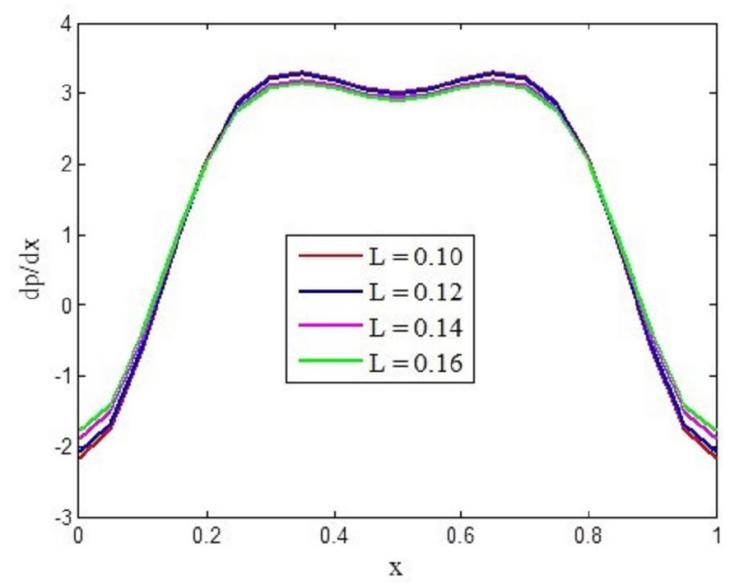

Fig. $16 \frac{d p}{d x}$ for $L$

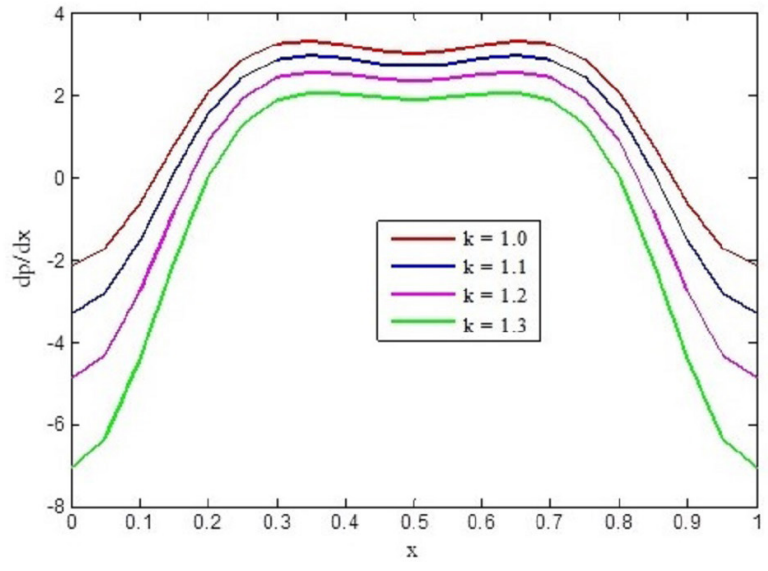

Fig. $17 \frac{d p}{d x}$ for $k$

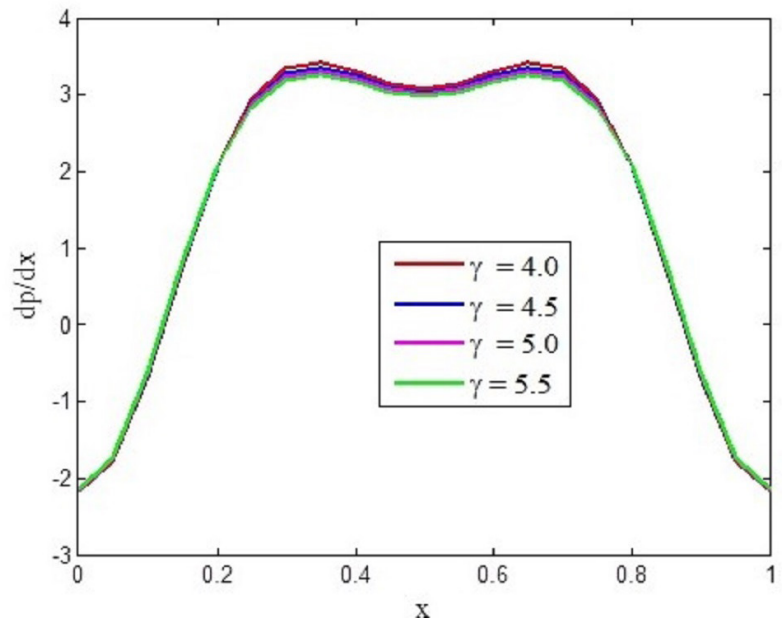

Fig. $18 \frac{d p}{d x}$ for $\gamma$

rate $\Theta$ to the effects of Hartmann number $M$, chemical reaction parameter $\gamma_{1}$, heat generation parameter $\beta$ and couple stress parameter $\gamma$. The pumping regions are peristaltic pumping are $\left(\Theta>0, \Delta P_{\lambda}>0\right)$, augment pumping $\left(\Theta<0, \Delta P_{\lambda}<0\right)$, retrograde pumping $\left(\Theta<0, \Delta P_{\lambda}>0\right)$, co-pumping $\left(\Theta>0, \Delta P_{\lambda}<0\right)$ and free pumping $\left(\Theta=0, \Delta P_{\lambda}=0\right)$. Fig. 19 is depicted that the pressure rise $\Delta P_{\lambda}$ depressing with an enhance in Hartmann number $M$ in the both peristaltic pumping region and free pumping region. Fig. 20 is observed that the dimensionless pressure rise per wave length $\Delta P_{\lambda}$ against the dimensionless average flux $\Theta$ to the influence of chemical reaction parameter $\gamma_{1}$. It is observed that the pressure rise decreases when the chemical reaction parameter $\gamma_{1}$ increases. Fig. 21 depicts quite the opposite nature of the effect of the heat generation parameter $\beta$. The influence of couple stress fluid parameter $\gamma$ on the pressure rise is decreases and which is elucidated from Fig. 22. 


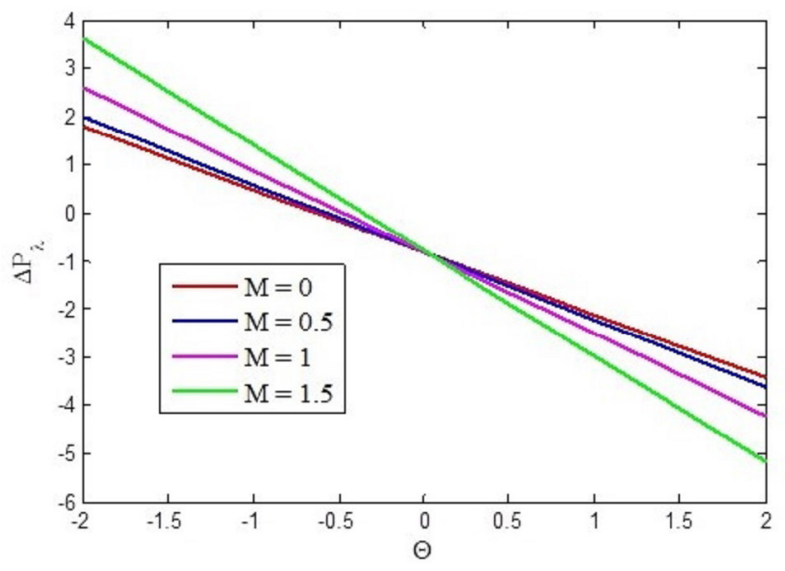

Fig. $19 \Delta P_{\lambda}$ for $M$

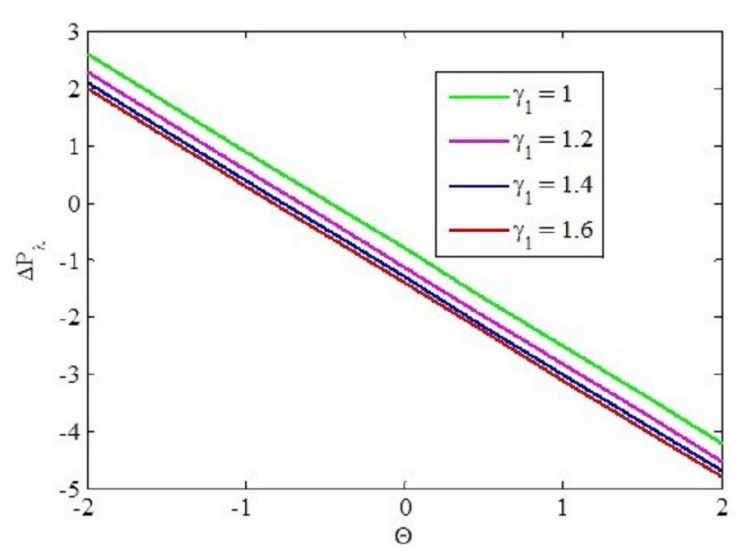

Fig. $20 \Delta P_{\lambda}$ for $\gamma_{1}$

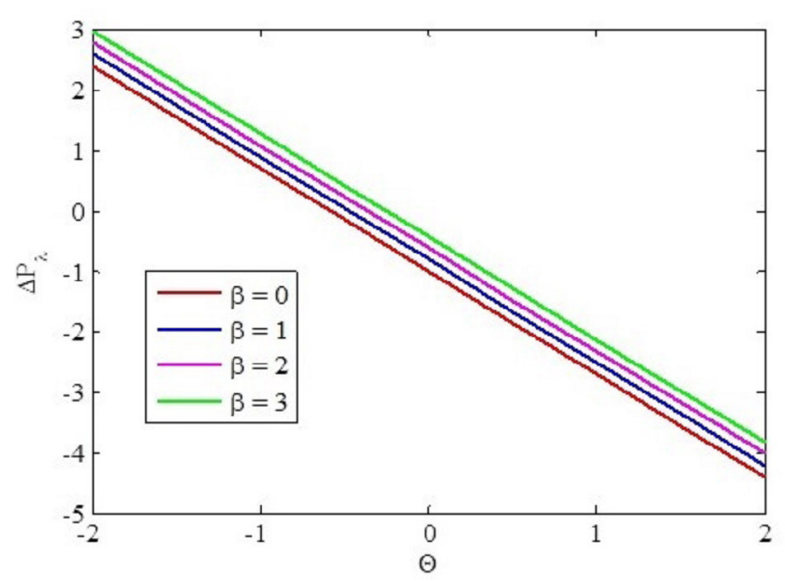

Fig. $21 \Delta P_{\lambda}$ for $\beta$

\subsection{Nusselt number}

Figs. 23 to 25 exhibit the influence of incorporated parameters such as Hartmann number $M$, Osmosis parameter $k$ and Joule heating parameter $A$ respectively on magnitude of Nusselt number. The heat transfer coefficient has oscillatory in nature due to peristaltic motion of walls. The heat transfer rate enhances for $M, k$ and $A$ from Figs. 23 to 25.

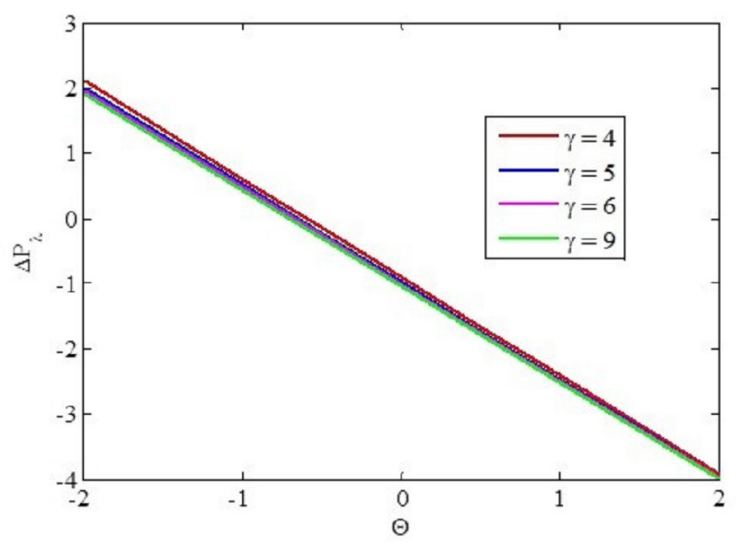

Fig. $22 \Delta P_{\lambda}$ for $\gamma$

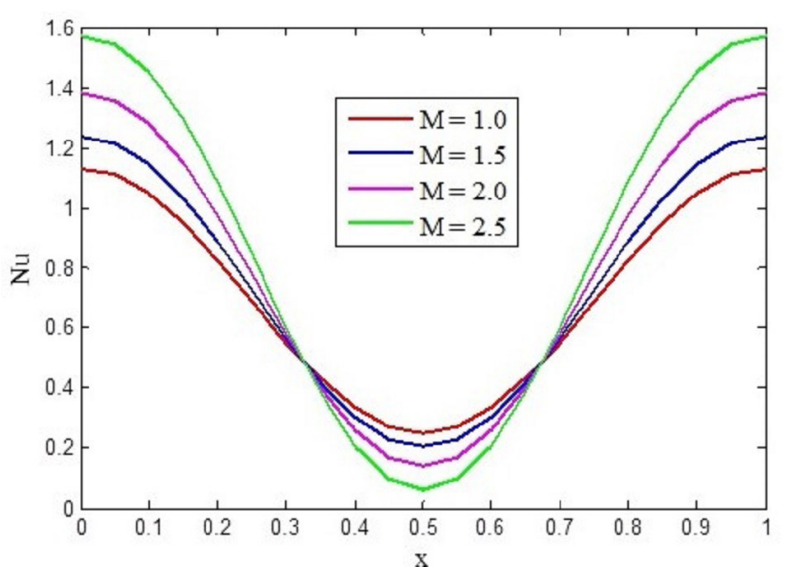

Fig. $23 \mathrm{Nu}$ for $M$

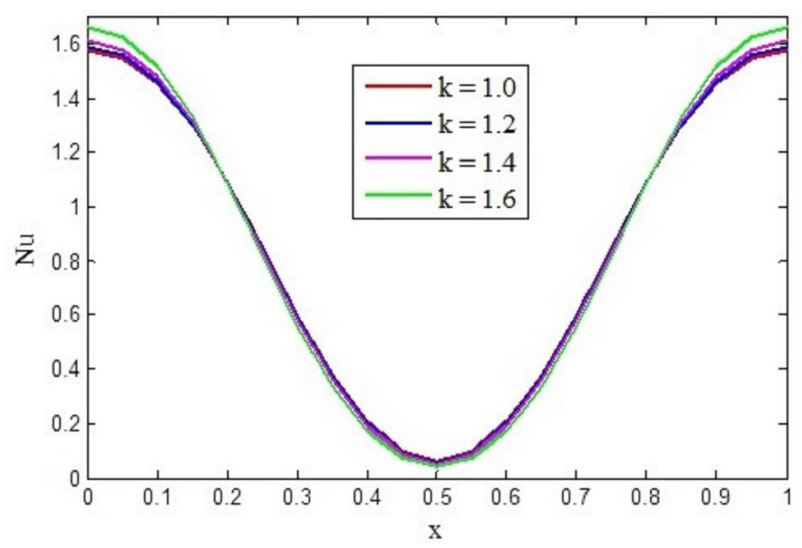

Fig. $24 \mathrm{Nu}$ for $k$

\subsection{Sherwood number}

The mass transfer coefficient shows the impact of different parameters of $\lambda_{1}$ and $k$ from Figs. 26 and 27. Fig. 26 depicts the Sherwood number depresses with the impact of $\lambda_{1}$ whereas it observes the mass transfer coefficient enhances as the parameter $k$ rises from Fig. 27. 


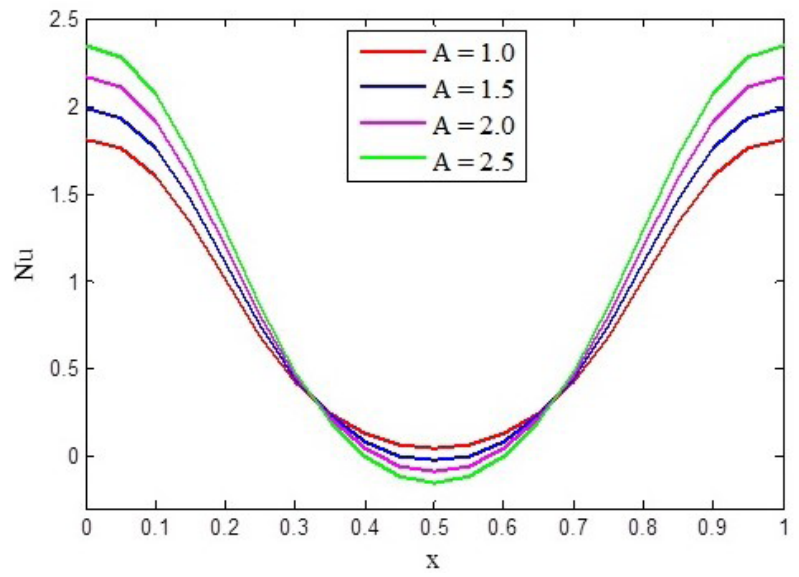

Fig. $25 \mathrm{Nu}$ for $A$

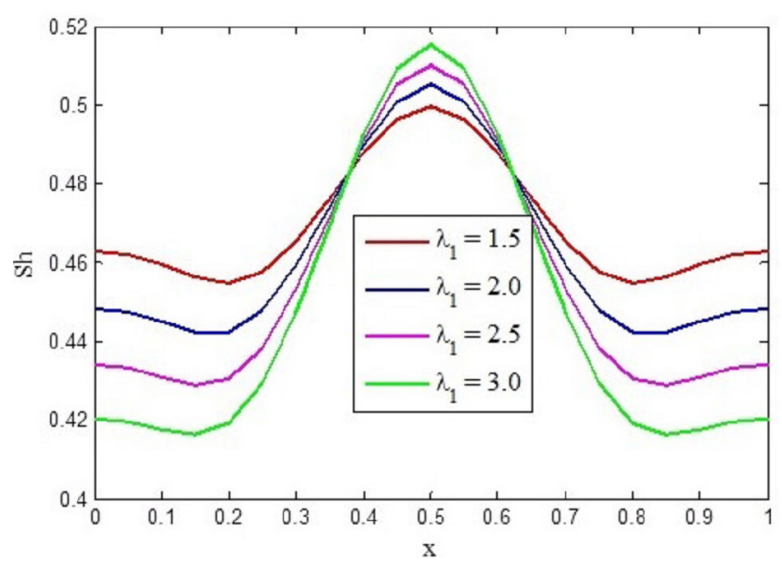

Fig. 26 Sh for $\lambda_{1}$

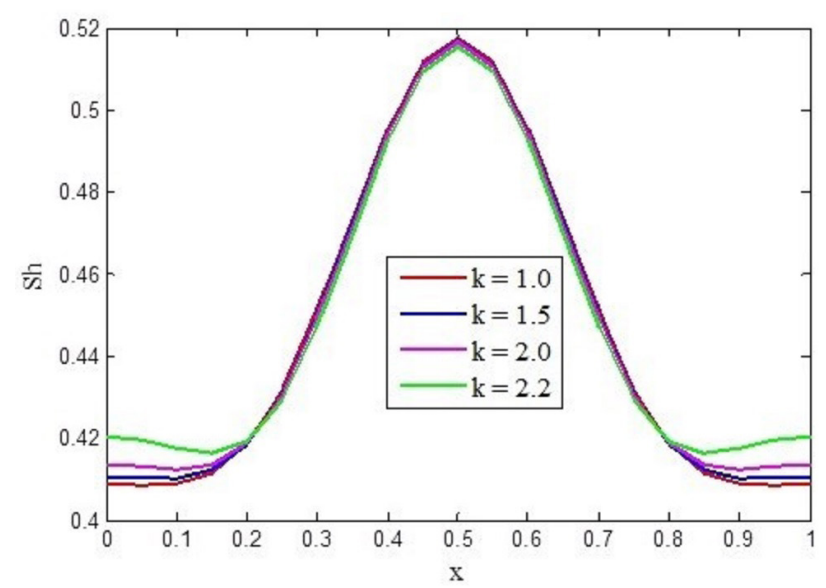

Fig. 27 Sh for $k$

\subsection{Trapping phenomenon}

The phenomenon of trapping in flow of fluid is trapping and is presented by drawing streamlines in the Figs. 28 to 31. A bolus is having by splitting of a streamline under important conditions and it is followed along with the wave in the wave frame. This process is called trapping. The bolus of trapping is observed to expand by enhancing $M$ from Figs. 28 to 29. However the size of bolus decreases by the rising effects of $\lambda_{1}$ as shown in Figs. 30 to 31 .

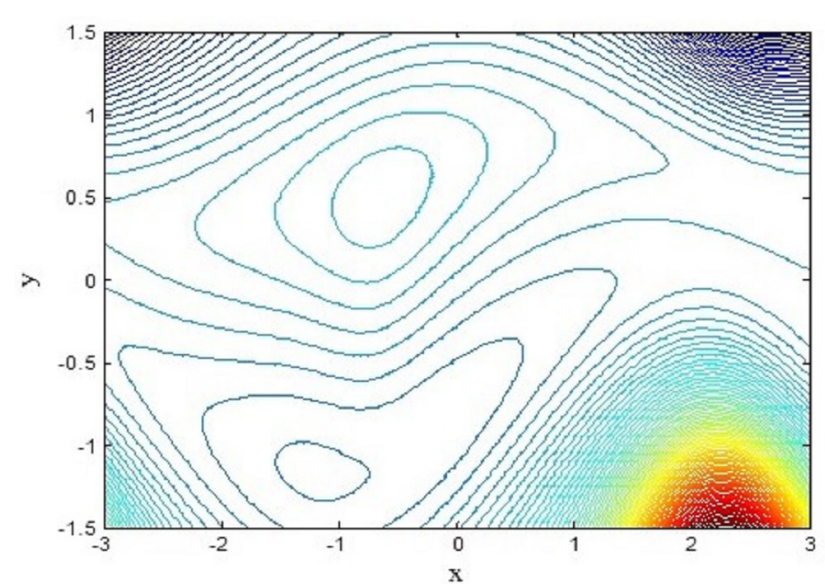

Fig. 28 Effect of $M$ on stream lines for $M=1.5$

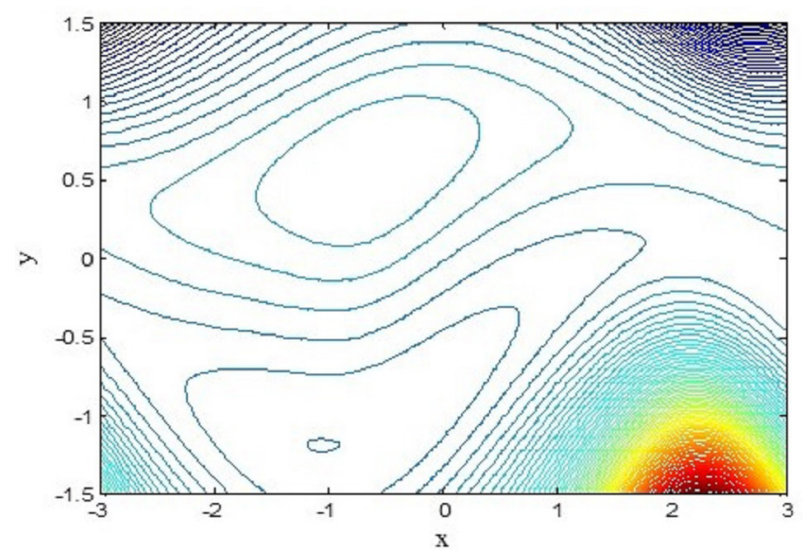

Fig. 29 Effect of $M$ on stream lines for $M=1.6$

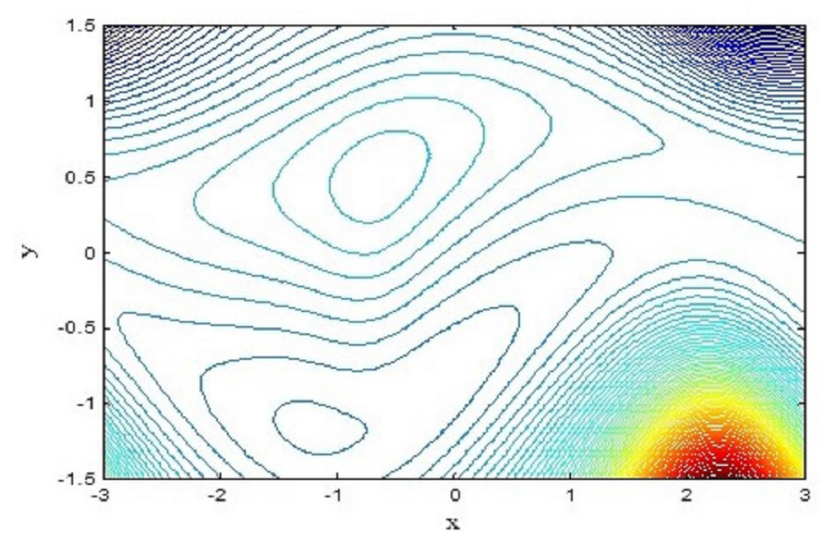

Fig. 30 Effect of $\lambda_{1}$ on stream lines for $\lambda_{1}=1.5$

The numerical results got by Mathematica software is validated with the Reddy et al. [26] and Hayat et al. [37]. To endorse the numerical results, Table 1 is shown for Nusselt number verses couple stress parameter. It is revealed that numerical results have a good agreement with the solutions of existing literature [26, 37] for the entire set of parametric values. 


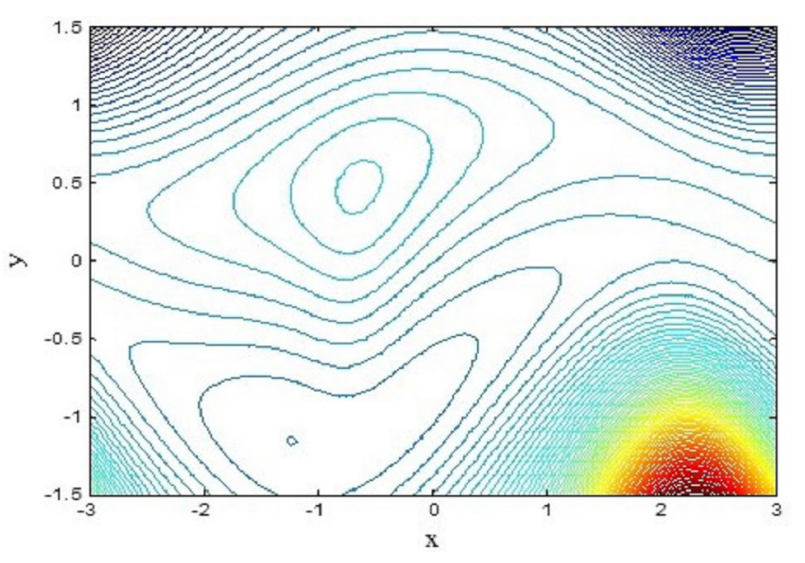

Fig. 31 Effect of $\lambda_{1}$ on stream lines for $\lambda_{1}=1.6$

Table 1 Comparison of values for Nusselt number

\begin{tabular}{cccc}
\hline & $\mathrm{Nu}$ & Reddy et al. [26] & Hayat et al. [37] \\
\hline 2 & -0.13297 & -0.13298 & -0.13298 \\
& -0.08374 & -0.083745 & -0.083745 \\
& -0.03412 & -0.034123 & -0.034123 \\
2.5 & -0.02528 & -0.025289 & -0.025289 \\
& 0.012862 & 0.012863 & 0.012863 \\
& 0.051613 & 0.051614 & 0.051614 \\
3 & -0.05475 & -0.054754 & -0.054754 \\
& -0.01328 & -0.013277 & -0.013277 \\
& 0.028704 & 0.028705 & 0.028705 \\
\hline
\end{tabular}

\section{Conclusion}

We have investigated the peristaltic transport of a heat and mass transfer of couple stress fluid on the combined impacts of electro-osmotically and pressure driven flow in an inclined asymmetric micro channel whose walls are varying sinusoidally with different wave trains. Effects of thermal radiation, chemical reaction and Joule heating have been accounted. The numerical solution for velocity, temperature distribution, concentration distribution and pumping characteristics are presented using small wave length and small Reynolds number. The important findings of present study are summarized as follows:

- The electro-osmotic flow of couple stress fluids in an inclined asymmetric channel is strongly depend on Debye length.

- Velocity diminishes with an enhance of $L$ and $\gamma$.

- Temperature rises with an strength of $A$ whereas depresses with an enhance of $R d$.

- Concentration decreases with enhance of $\gamma_{1}$.

- It is observed that pressure gradient has oscillatory behavior.

- Pressure rise decreases with an effect of increasing $\gamma$.

- The absence of electro-osmosis, our results are in good agreement with Reddy et al. [26].
- As the couple stress parameter increases, axial velocity increases in core part of the channel.

- Behaviors of axial velocity component and temperature profile are qualitatively similar for Hartmann number.

\section{Nomenclature}

\section{Symbol Description}

$\left(a_{1}^{\prime}, b_{2}^{\prime}\right)$ Amplitude of left and right of the micro asymmetric vessel (m)

$(a, b)$ Dimensionless amplitude of left and right of the micro asymmetric vessel

c Wave speed $(\mathrm{m} / \mathrm{s})$

$c_{p} \quad$ Specific heat capacity $(\mathrm{J} /(\mathrm{kgK}))\left(\operatorname{Kelvin}^{-1} \mathrm{sec}^{-2} \mathrm{~m}^{2}\right)$

$d \quad$ Half channel width (m)

$E_{x} \quad$ Applied electrical field

$\bar{E} \quad$ Electric potential

E Dimensionless electric potential

e $\quad$ Fundamental charge

$F \quad$ Dimensionless mean flows

Gr Thermal Grashof number

$g \quad$ Acceleration due to gravity $\left(\mathrm{m} \mathrm{s}^{-1}\right)$

$\left(h_{1}, h_{2}\right)$ Heat transfer coefficients for left and right walls $\left(\mathrm{kg} \mathrm{sec}^{-3}\right.$ Kelvin $\left.^{-1}\right)$

$k^{\prime} \quad$ Permeability of the porous walls (Darcy number) (W/m Kelvin)

$k^{*} \quad$ Mean absorption coefficient $\left(\mathrm{m}^{-1}\right)$

$L \quad$ Slip parameter

$\bar{p} \quad$ Dimensional pressures $\left(\mathrm{kg} / \mathrm{m} \mathrm{s}^{2}\right)$

$P \quad$ Dimensionless pressure

Pr Prandtl number

$\bar{q}_{r} \quad$ Uni-directional thermal radiative flux

$\bar{Q}_{0} \quad$ Constant viscous dissipation function $\left(\mathrm{kg} \mathrm{m}^{-1} \mathrm{sec}^{-3}\right)$

$R n \quad$ Thermal radiation parameter

Re Reynolds number

$t \quad$ Dimensional time (sec)

$\bar{T} \quad$ Temperature (Kelvin)

$T_{m} \quad$ Average temperature (Kelvin)

$\left(T_{0}, T_{1}\right)$ Temperature at the left and right walls respectively (Kelvin)

$\left(u^{*}, v^{*}\right)$ Wave frame velocity components of $((X, Y), m)$ $\left(\mathrm{m} \mathrm{sec}^{-1}\right)$

$(U, V) \quad$ Fixed frame velocity components of $(x, y)$

$(u, v) \quad$ Dimensionless velocity components of $(x, y)$

\section{Greek symbols}

$\bar{\rho}_{e} \quad$ Electric charge density per unit volume

$\mu \quad$ Fluid dynamic viscosity $\left(\mathrm{kg} \mathrm{m}^{-1} \mathrm{sec}^{-1}\right)$

$\gamma_{v} \quad$ Spin gradient viscosity $\left(\mathrm{kg} \mathrm{m} \mathrm{sec}^{-1}\right)$ 
$k_{v} \quad$ Kinematic rotational viscosity $\left(\mathrm{m}^{2} \mathrm{sec}^{-1}\right)$

$\lambda \quad$ Wavelength (m)

$\kappa_{e f} \quad$ Thermal conductivity $(\mathrm{W} /(\mathrm{m} \mathrm{K}))$,

$\sigma^{*} \quad$ Stefan-Boltzmann constant $\left(\mathrm{W} \mathrm{m}^{-2} \mathrm{Kelvin}^{-4}\right)$

$\varepsilon \quad$ Dielectric permittivity of the medium $\left(\mathrm{F} \mathrm{m}^{-1}\right)$

$\zeta \quad$ Zeta potential (v)

$\phi \quad$ Phase difference

$\sigma \quad$ Dimensionless solute concentration

$\alpha_{m} \quad$ Thermal diffusivity

\section{References}

[1] Tikhomolova, K. P. "Electro-Osmosis", Ellis Horwood, London, UK, 1993.

[2] Ginsburg, H. "Analysis of plant root electropotentials", Journal of Theoretical Biology, 37(3), pp. 389-412, 1972. https://doi.org/10.1016/0022-5193(72)90082-3

[3] Sansalone, V., Kaiser, J., Naili, S., Lemaire, T. "Interstitial fluid flow within bone canaliculi and electro-chemo-mechanica features of the canalicular milieu", Biomechanics and Modeling in Mechanobiology, 12(3), pp. 533-553, 2013. https://doi.org/10.1007/s10237-012-0422-7

[4] Marshall, E. A. "The osmotic flow of an electrolyte through a charged porous membrane", Journal of Theoretical Biology, 66(1), pp. 107-135, 1977. https://doi.org/10.1016/0022-5193(77)90315-0

[5] Grimnes, S. "Skin impedance and electro-osmosis in the human epidermis", Medical and Biological Engineering and Computing, 21(6), pp. 739-749, 1983.

https://doi.org/10.1007/BF02464037

[6] Davis, T. A. "Electrodialysis", William Andrew Publisher, Noyes, NJ, USA, 1990.

[7] Orsat, V., Raghavan, V. G. S., Norris, E. R. "Food processing waste dewatering by electro-osmosis", Canadian Agricultural Engineering, 38(1), pp. 63-67, 1996.

[8] Manz, A., Effenhauser, C. S., Burggraf, N., Harrison. D. J., Seiler, K., Fluri, K. "Electroosmotic pumping and electrophoretic separations for miniaturized chemical analysis systems", Journal of Micromechanics and Microengineering, 4(4), pp. 257-265, 1994. https://doi.org/10.1088/0960-1317/4/4/010

[9] Ngoma, G. D., Erchiqui, F. "Pressure gradient and electroosmotic effects on two immiscible fluids in a microchannel between two parallel plates", Journal of Micromechanics and Microengineering, 16(1), pp. 83-91, 2005.

https://doi.org/10.1088/0960-1317/16/1/012

[10] Zhao, T. S., Liao, Q. "Thermal effects on electro-osmotic pumping of liquids in microchannels", Journal of Micromechanics and Microengineering, 12(6), pp. 962-970, 2002. https://doi.org/10.1088/0960-1317/12/6/329

[11] Stokes, V. K. "Couple stresses in fluids", The Physics of Fluids, 9(9), pp. 1709-1715, 1966. https://oi.org/10.1063/1.1761925

[12] Devakar, M., Iyengar, V. "Stokes' Problems for an Incompressible Couple Stress Fluid", Nonlinear Analysis: Modelling and Control, 13(2), pp. 181-190, 2008.

https://doi.org/10.15388/NA.2008.13.2.14578 $\alpha^{\prime} \quad$ Slip coefficient at the surface of the porous walls

$\beta \quad$ Non-dimensional heat source/sink parameter

$\Theta \quad$ Dimensionless time average flux

$\gamma \quad$ Rescaled nano particle volume fraction

$\kappa \quad$ Electro-osmotic parameter (Debye length)

$\Omega \quad$ Axial micro rotation velocity

$\theta \quad$ Dimensionless temperature

$\psi \quad$ Stream function

$\delta \quad$ Wave number

[13] Duan, J. S., Qui, X. "Stokes' second problem of viscoelastic fluids with constitutive equation of distributed-order derivative", Applied Mathematics and Computation, 331, pp. 130-139, 2018. https://doi.org/10.1016/j.amc.2018.02.028

[14] Ramesh, K. "Influence of heat and mass transfer on peristaltic flow of couple stress fluid through porous medium in the presence of inclined magnetic field in an inclined asymmetric channel", Journal of Molecular Liquids, 219, pp. 256-271, 2016. https://doi.org/10.1016/j.molliq.2016.03.010

[15] Alsaedi, A., Ali, N., Tripathi, D., Hayat, T. "Peristaltic flow of couple stress fluid through uniform porous medium", Applied Mathematics and Mechanics, 35(4), pp. 469-480, 2014. https://doi.org/10.1007/s10483-014-1805-8

[16] Kaladhar, K. "Natural Convection Flow of Couple Stress Fluid in a Vertical Channel With Hall and Joule Heating Effects", Procedia Engineering, 127, pp. 1071-1078, 2015.

https://doi.org/10.1016/j.proeng.2015.11.465

[17] Hayat, T., Iqbal, M., Yasmin, H., Alsaadi, F. "Hall effects on peristaltic flow of couple stress fluid in an inclined asymmetric channel", International Journal of Biomathematics, 7(5), Article Number: 1450057, 2014.

https://doi.org/10.1142/S1793524514500570

[18] Latham, T. W. "Fluid motion in a peristaltic pump", Master's Thesis, Massachusetts Institute of Technology, 1966.

[19] Shapiro, A. H., Jaffrin, M. Y., Weinberg, S. L. "Peristaltic pumping with long wavelengths at low Reynolds numbers", Journal of Fluid Mechanics, 37(4), pp. 799-825, 1969. https://doi.org/10.1017/S0022112069000899

[20] Mekheimer, K. S., Elmaboud, Y. A. "Peristaltic flow of a couple stress fluid in an annulus: Application of an endoscope", Physica A: Statistical Mechanics and its Applications, 387(11), pp. 2403-2415, 2008. https://doi.org/10.1016/j.physa.2007.12.017

[21] Makinde, O. D., Mhone, P. "Hydromagnetic Effects on Internal Flow Separation in a Diverging Channel", Romanian Journal of Physics, 51(9-10), pp. 959-966, 2006.

[22] Hayat, T., Javed, M., Ali, N. "MHD Peristaltic Transport of Jeffrey Fluid in a Channel With Compliant Walls and Porous Space", Transport in Porous Media, 74(3), pp. 259-274, 2008. https://doi.org/10.1007/s11242-007-9196-2

[23] Adesanya, S. O., Makinde, O. D. "Heat Transfer to Magnetohydrodynamic Non-Newtonian Couple Stress Pulsatile Flow Between two Parallel Porous Plates", Zeitschrift for Naturforschung 67(10-11), pp. 647-656, 2014. https://doi.org/10.5560/ZNA.2012-0073 
[24] Adesanya, S. O., Makinde, O. D. "Irreversibility analysis in a couple stress film flow along an inclined heated plate with adiabatic free surface", Physica A: Statistical Mechanics and its Applications, 432, pp. 222-229, 2015.

https://doi.org/10.1016/j.physa.2015.02.062

[25] Reddy, M. G., Reddy, K. V. "Impact of velocity slip and joule heating on MHD peristaltic flow through a porous medium with chemical reaction", Journal of the Nigerian Mathematical Society, 35(1), pp. 227-244, 2016.

https://doi.org/10.1016/j.jnnms.2016.02.005

[26] Reddy, M. G., Reddy, K. V., Makinde, O. D. "Hydromagnetic peristaltic motion of a reacting and radiating couple stress fluid in an inclined asymmetric channel filled with a porous medium", Alexandria Engineering Journal, 55(2), pp. 1841-1853, 2016. https://doi.org/10.1016/j.aej.2016.04.010

[27] Mishra, M., Rao, A. R. "Peristaltic transport of a Newtonian fluid in an asymmetric channel", Zeitschrift für angewandte Mathematik und Physik ZAMP, 54(3), pp. 532-550, 2003. https://doi.org/10.1007/s00033-003-1070-7

[28] Kothandapani, M., Srinivas, S. "Peristatltic transport of a Jeffrey fluid under the effect of magnetic field in an asymmetric channel", International Journal of Non-Linear Mechanics, 43(9), pp. 915-924, 2008. https://doi.org/10.1016/j.ijnonlinmec.2008.06.009

[29] Abbasi, F. M., Hayat, T., Ahmad, B., Chen, B. "Peristaltic flow with convective mass condition and thermal radiation", Journal of Central South University, 22(6), pp. 2369-2375, 2015. https://doi.org/10.1007/s11771-015-2762-9

[30] Reddy, M. G., Reddy, K. V., Makinde, O. D. "Heat Transfer on MHD Peristaltic Rotating Flow of a Jeffrey Fluid in an Asymmetric Channel", International Journal of Applied and Computational Mathematics, 3(4), pp. 3201-3227, 2017. https://doi.org/10.1007/s40819-016-0293-1
[31] Makinde, O. D., Reddy, M. G., Reddy, K. V. "Effects of Thermal Radiation on MHD Peristaltic Motion of Walters-B Fluid with Heat Source and Slip Conditions", Journal of Applied Fluid Mechanics, 10(4), pp. 1105-1112, 2017.

https://doi.org/10.18869/acadpub.jafm.73.241.27082

[32] Reza, J., Mebarek-Oudina, F., Makinde, O. D. "MHD Slip Flow of $\mathrm{Cu}-$ Kerosene Nanofluid in a Channel with Stretching Walls Using 3-Stage Lobatto IIIA Formula", Defect and Diffusion Forum, 387, pp. 51-62, 2018.

https://doi.org/10.4028/www.scientific.net/DDF.387.51

[33] Marzougui, S., Mebarek-Oudina, F., Assia, A., Magherbi, M., Shah, Z., Ramesh, K. "Entropy generation on magnetoconvective flow of copper-water nanofuid in a cavity with chamfers", Journal of Thermal Analysis and Calorimetry, 2020. https://doi.org/10.1007/s10973-020-09662-3

[34] Raza, J., Mebarek-Oudina, F., Ram, P., Sharma, S. "MHD Flow of Non-Newtonian Molybdenum Disulfide Nanofluid in a Converging/Diverging Channel with Rosseland Radiation", Defect and Diffusion Forum, 401, pp. 92-106, 2020. https://doi.org/10.4028/www.scientific.net/DDF.401.92

[35] Mebarek-Oudina, F., Aissa, A., Mahanthesh, B., Öztop, H. F. "Heat transport of magnetized Newtonian nanoliquids in an annular space between porous vertical cylinders with discrete heat source", International Communications in Heat and Mass Transfer, 117, Article Number: 104737, 2020. https://doi.org/10.1016/j.icheatmasstransfer.2020.104737

[36] Khan, U., Zaib, A., Mebarek-Oudina, F. "Mixed Convective Magneto Flow of $\mathrm{SiO}_{2}-\mathrm{MoS}_{2} / \mathrm{C}_{2} \mathrm{H}_{6} \mathrm{O}_{2}$ Hybrid Nanoliquids Through a Vertical Stretching/Shrinking Wedge: Stability Analysis", Arabian Journal for Science and Engineering, 45(11), pp. 9061-9073, 2020. https://doi.org/10.1007/s13369-020-04680-7

[37] Hayat, T., Asghar, S., Tanveer, A., Alsaedi, A. "Chemical reaction in peristaltic motion of MHD couple stress fluid in channel with Soret and Dufour effects", Results in Physics, 10, pp. 69-80, 2018. https://doi.org/10.1016/j.rinp.2018.04.040 